\title{
ESTIMATION OF SOME GENETIC PARAMETERS USING LINE $\times$ TESTER ANALYSIS OF COMMON WHEAT (TRITICUM AESTIVUM L.)
}

\author{
HAMA-AMIN, T. N. ${ }^{*}-$ TOWFIQ, S. I. \\ Crop Science Department, College of Agricultural Sciences, University of Sulaimani \\ Sulaimani, Iraq \\ *Corresponding author \\ e-mail: taban.najmaddin@univsul.edu.iq,tabantaby@yahoo.com \\ (Received $19^{\text {th }}$ Mar 2019; accepted $13^{\text {th }}$ Jun 2019)
}

\begin{abstract}
Seven common wheat (Triticum aestivum L.) cultivars were crossed in a line×tester matting design. The $12 \mathrm{~F}_{1}$ and their parents were evaluated using a Completely Randomized Block Design (CRBD) replicated three times in Kurdistan Reign-Iraq at two different locations, first Kanipanka in Sharazoor Valley, and the second was Qlyasan Agricultural Research Station, University of Sulaimani, during the winter season of 2017-2018. The Least Significant Differences Test (LSD) at (P $\leq 0.05)$ was carried out to compare the means of the characters. The results confirmed that sufficient genetic variability was included with all traits studied. The cross Aras $\times$ Iba- 95 at Kanipanka location and Klal $\times$ Cham-6 at Qlyasan location had better grain yield/plant mean than their parents. The cross Aras $\times$ Iba- 95 and Klal $\times$ Cham- 6 at both locations exhibited maximum positive heterosis for grain yield/plant and most of their components. Line parents Aras and Klal are good general combiners for grain yield/plant and most its components at both locations respectively. The cross Aras $\times$ Iba- 95 is the better specific combiner to increase grain yield/plant at both sites. The superiority of non-additive type of gene actions in controlling the inheritance of these characters was confirmed.
\end{abstract}

Keywords: heterosis, combining ability, heritability, gene action

\section{Introduction}

Bread wheat (Triticum aestivum L.) is the most important crop being one-fifth of the total calories for the population of the world (Sehgal et al., 2015). Wheat cultivation represents approximately $19 \%$ of global main cereal crop production (Todorovska et al., 2009). Considerable cultivated species of wheat include: Triticum aestivum, which is a hexaploid species and is widely seeded in the world (Moon, 2008). It has been imposed that the world population would increase to over 9 billion by the year 2050 (United Nations, Population Division, 2015). The population growth will increase the request for wheat crop by $60 \%$ compared with current years. To meet this demand, global annual yield increases have to rise from the current level of $1 \%$ per year to $1.6 \%$ per year until 2050 (Wheat, 2014). For this cause, the breeders have to share demanding need to increase yield potential of wheat by creating new wheat varieties with likable genetic makeup (Erkul et al., 2010). This could be reached by searching maximum genetic potential from available wheat germplasm (Khan et al., 2007). Common wheat is one of the earliest domesticated crops. It is a prime staple food for around $40 \%$ of the world's population (Kuzmanovic et al., 2014). Wheat provides about $20 \%$ of the calorific input for the world population and, for cereal crops has the third highest global production after maize and rice (FAO, 2014). Wheat is widely cultivated in various regions of the world, being well suited for growth in area between latitudes of $30^{\circ}$ and $60^{\circ} \mathrm{N}$ and $27^{\circ}$ and $40^{\circ} \mathrm{S}$ (Curtis, 2002). 349 million 
tons of this crop was harvested in 2016, and more than 220 million hectare of the world was cultivated by wheat. Due to the raising of the world population, wheat plays a main role in the national economy of developing countries. Read wheat is one of the major food grain crops in the world, as it provides $20 \%$ of the total energy and protein in the human diet (FAO, 2016). Most of wheat planted areas are located in arid or semi-arid regions where a biotic stresses, especially drought stress, are a main constraint for wheat production (Tahmasebi et al., 2014). Current wheat production does not meet global demand due to the declining arable land area, increasing global population, and the effects of climate condition change on the environment (Tester and Langridge, 2010; Altieri and Nicholls, 2017). To meet the need for population growth, the country's wheat requirement by 2030 has been estimated at 100 million metric tons and to reach this goal, wheat productivity has to be increased at the rate of $<1$ per annum and this can be fulfilled through horizontal approach i.e. by rising area under cultivation or through varietal/hybrid improvement, which is one of the substantial tool to take a volume jump in production and productivity under various agro- climatic conditions (Rahul, 2017). Developing hybrid wheat varieties with desirable traits require a thorough knowledge about the existing genetic variability (Maniee et al., 2009; Kahrizi et al., 2010a,b). The more the genetic diverse parents, the greater the chances of obtaining higher heterotic expression in $F_{1} s$ and broad spectrum of variability in segregating population (Shekhawat et al., 2001). The line $\times$ tester analysis method proposed by Kempthorne (1957) is one of the powerful tools available to evaluate the combining ability effects and aids in selecting desirable parents and crosses for exploitation in pedigree breeding (Basbag, 2007; Jain and Sastry, 2012; Rashid et al., 2013). Performances itself do not necessarily reveal which parents are best or weak combiners. To get up this difficulty, it is necessary to collect information on the nature of gene effects. General combining ability is assigned to additive type of gene effects, while specific combining ability is attributed to nonadditive of gene works. Non-additive gene type of actions is not reliably fixable whereas additive type of gene effects or complementary type epistatic gene interactions is reliably fixable (Xiang and Li, 2001; Yan and Hunt, 2002; Iqbal et al., 2007). The objectives of this study are to estimate the combining ability, to estimate the nature and magnitude of gene actions, to evaluate heterosis and heritability for yield and yield related traits in a line $\times$ tester method in bread wheat.

\section{Materials and methods}

This study was carried out at two different locations, in Kurdistan Region-Iraq. First

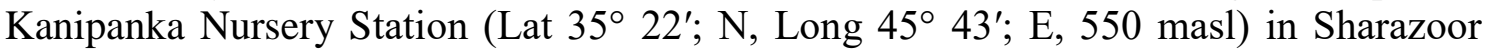
Valley $35 \mathrm{~km}$ East of Sulaimani City, and second was at Qlyasan Agricultural Research Station, College of Agricultural Sciences-University of Sulaimani located (Lat $35^{\circ} 34^{\prime}$ 307"; N, Long 45 21' 992"; E, 765 masl) 2 km North West of Sulaimani City, during 2017-2018. The experimental material comprises seven bread wheat (Triticum aestivum L.) genotypes. Four genotypes (Aras, Hasad, Kauz and Klal) were used as females, hereafter designated as lines, and three genotypes (Iba-95, Cham-6 and Saber beg) were used as males, fixed as testers. The seven parents were crossed to produce $12 \mathrm{~F}_{1}$ crosses according to the line $\times$ tester mating design developed by Kempthorne (1957). $F_{1}$ seeds were sown in the field, along with their parents, in Complete Randomize Block Design (CRBD) with three replication. Each plot comprised one row of $2 \mathrm{~m}$ length with space 
of $40 \mathrm{~cm}$ between rows and seeds were placed $15 \mathrm{~cm}$ apart. Five competitive plants (excluding border plants) were chosen and data were recorded for:

1-Evaluated traits:

Number of spikes/plant, Weight of spikes/plant (g), Average spike weight (g), Spike length $(\mathrm{cm})$, Number of grains/spike, Weight of grains/spike $(\mathrm{g}), 1000$-grain weight $(\mathrm{g})$, Biological yield/plant, Grain yield/plant and Harvest index.

2-Genetic parameters:

General Combining Ability (gca) and effects, Specific Combining Ability (sca) and effects, Heterosis percentage as the $F_{1} s$ deviation from average parental values, Heritability in Broad Sense, Heritability in Narrow Sense, Average Degree of Dominance $(\bar{a})$.

3-Association analysis and path coefficient analysis.

The collected data were submitted to analysis of variance as proposed by Steel and Torrie (1980) to estimate significant differences among genotypes. Combining ability effects are very important genetic parameters in determining the next phase of breeding programs. They were computed according to the linextester method (Singh and Chaudhary, 1985).

\section{Climate conditions of Sulaimani region}

The climate of Sulaimani governorate is semi-arid environment: wet and cold in winter dry and hot in summer; During July and August, the average temperature is between $39-43^{\circ} \mathrm{C}$, and overwhelmingly amount to nearly $50^{\circ} \mathrm{C}$. Autumn means high temperatures are $20-30^{\circ} \mathrm{C}$ in October, cooling slightly in November. Precipitation is limited to winter and spring months, and the overall average annual rainfall of 550-700 mm was at Sulaimani city. An overview of experimental conditions is given in Table 1 .

Table 1. The meteorological data of the two locations

\begin{tabular}{|c|c|c|c|c|c|c|c|c|}
\hline \multirow[b]{2}{*}{ Month } & \multicolumn{4}{|c|}{ Kanipanka location } & \multicolumn{4}{|c|}{ Qlyasan location } \\
\hline & $\begin{array}{c}\text { Min } \\
\text { Temp. }\left(C^{\circ}\right) \\
\end{array}$ & $\begin{array}{c}\text { Max } \\
\text { Temp. }\left(C^{\circ}\right) \\
\end{array}$ & $\begin{array}{c}\text { Avg. } \\
\text { Temp.(C })\end{array}$ & $\begin{array}{c}\text { Rainfall } \\
(\mathbf{m m})\end{array}$ & \begin{tabular}{c|} 
Min \\
Temp. $\left(C^{\circ}\right)$ \\
\end{tabular} & \begin{tabular}{c|} 
Max \\
Temp. $\left(C^{\circ}\right)$ \\
\end{tabular} & $\begin{array}{c}\text { Avg. } \\
\text { Temp.(C }\end{array}$ & $\begin{array}{c}\text { Rainfall } \\
(\mathbf{m m})\end{array}$ \\
\hline October & 22.6 & 30.0 & 15.1 & - & 10.4 & 33.1 & 21.2 & 10.0 \\
\hline November & 14.4 & 20.0 & 8.8 & 71 & 7.6 & 23.9 & 14.2 & 114.6 \\
\hline December & 10.2 & 16.1 & 4.4 & 18.5 & -2.5 & 17.8 & 7.0 & 22.2 \\
\hline January & 7.8 & 12.5 & 3.1 & 60 & 1.4 & 15.6 & 7.8 & 72.4 \\
\hline February & 10.3 & 14.9 & 6.1 & 281 & -2.3 & 20.9 & 8.7 & 323.0 \\
\hline March & 14.7 & 21.3 & 8.1 & 19 & 1.0 & 24.4 & 13.0 & 44.6 \\
\hline April & 17.1 & 24 & 10.5 & 90.5 & 2.2 & 31.6 & 17.4 & 98.6 \\
\hline May & 22.2 & 29.5 & 15.0 & 68 & 13.0 & 38.1 & 24.7 & 70.4 \\
\hline $\begin{array}{l}\text { Total } \\
\text { rainfall }\end{array}$ & & & & 608 & & & & 755.8 \\
\hline
\end{tabular}

\section{Soil analysis}

The soil samples for both locations were taken from both sites in Sulaimani governorate in Kurdistan region, Iraq. It was obtain from surface $(0-30 \mathrm{~cm})$, and air dried which ground to pass through a 2-mm sieve prior to analysis, shown in Table 2. 
Table 2. Some physicochemical properties of the soil samples for locations of the experiment

\begin{tabular}{|c|c|c|c|c|c|c|c|}
\hline \multirow{2}{*}{ Location } & \multicolumn{7}{|c|}{ Physical properties of the studied soil / Particle size distribution (PSD) g/kg } \\
\hline & \multicolumn{2}{|c|}{ Sand } & Silt & Clay & \multicolumn{3}{|c|}{ Texture class } \\
\hline \multirow{5}{*}{$\begin{array}{c}\text { Kanipanka } \\
\text { Qlyasan }\end{array}$} & \multirow{2}{*}{\multicolumn{2}{|c|}{$\begin{array}{c}214.00 \\
90.40\end{array}$}} & \multirow{2}{*}{$\begin{array}{l}540.00 \\
508.40 \\
\end{array}$} & \multirow{2}{*}{$\begin{array}{l}246.00 \\
401.20 \\
\end{array}$} & \multirow{2}{*}{\multicolumn{3}{|c|}{$\begin{array}{l}\text { Salty loam } \\
\text { salty clay }\end{array}$}} \\
\hline & & & & & & & \\
\hline & \multicolumn{7}{|c|}{ Chemical properties of the studied soil } \\
\hline & \multirow{2}{*}{$\begin{array}{c}\mathrm{pH} \\
\mathrm{dS} \mathrm{m}^{-1}\end{array}$} & \multirow{2}{*}{$\begin{array}{c}\mathrm{EC}_{\mathrm{e}} \\
\mathrm{g} \mathrm{kg}^{-1}\end{array}$} & \multirow{2}{*}{$\begin{array}{c}\mathrm{OM} \\
\mathrm{Cmol}_{\mathrm{c}} \mathrm{kg}^{-1}\end{array}$} & \multirow{2}{*}{$\begin{array}{c}\mathrm{CEC} \\
\mathrm{mg} \mathrm{kg}^{-1}\end{array}$} & \multirow{2}{*}{$\begin{array}{c}\text { Available } \\
\text { P }\end{array}$} & \multicolumn{2}{|c|}{$\begin{array}{c}\mathrm{CaCO}_{3} \text { equivalent } \\
\mathrm{g} \mathrm{kg}^{-1}\end{array}$} \\
\hline & & & & & & Total & Active \\
\hline Kanipanka & 8.05 & 0.16 & 22.03 & 22.10 & 7.44 & 195.00 & 100.00 \\
\hline \multirow[t]{3}{*}{ Qlyasan } & 7.80 & 0.38 & 16.06 & 29.76 & 9.61 & 230.00 & 117.00 \\
\hline & \multicolumn{7}{|c|}{ Soluble ions mmol $\mathrm{L}^{-1}$} \\
\hline & $\mathrm{Ca}^{2+}$ & $\mathrm{Mg}^{2+}$ & $\mathrm{Na}^{+}$ & $\mathrm{K}^{+}$ & $\mathrm{HCO}_{3}^{-}$ & $\mathrm{Cl}^{-}$ & $\mathrm{SO}_{4}{ }^{2-}$ \\
\hline Kanipanka & 1.20 & 1.05 & 0.19 & 0.05 & 3.20 & 0.90 & 0.91 \\
\hline \multirow[t]{3}{*}{ Qlyasan } & 2.20 & 1.80 & 0.10 & 0.13 & 2.34 & 0.80 & 0.88 \\
\hline & \multicolumn{7}{|c|}{ Available micronutrients mg kg-1 } \\
\hline & \multicolumn{2}{|c|}{$\mathrm{Zn}$} & & \multicolumn{2}{|l|}{$\mathrm{Cu}$} & \multicolumn{2}{|l|}{$\mathrm{Fe}$} \\
\hline Kanipanka & \multirow{2}{*}{\multicolumn{2}{|c|}{$\begin{array}{l}1.563 \\
0.450\end{array}$}} & & \multirow{2}{*}{$\begin{array}{l}5.07 \\
4.96\end{array}$} & & \multicolumn{2}{|l|}{5.15} \\
\hline Qlyasan & & & & & & 3.23 & \\
\hline
\end{tabular}

\section{Results}

The analysis of variance ratified highly significant genotype effect for all characters studied. This confirmed the presence of enough genetic variability among genotypes at both locations. The mean squares for parents was highly significant for number of spikes/plant, average spike weight, biological yield/plant and harvest index, but it was significant for spike length, number of grains/spike, weight of grains/spike and 1000-grain weight, while for other characters it was not significant at the first location. At the second location the mean squares for parents was highly significant for all characters except biological yield/plant, grain yield/plant and harvest index which was not significant. The mean squares due to crosses were highly significant for all characters at both locations with the exception of number of spikes/plant and average spike weight which was significant at the second location (Table 3).

The mean performance of parents and $\mathrm{F}_{1} \mathrm{~s}$ crosses for all characters were represented in Table 4. At the first location the cross Aras $\times$ Iba-95 recorded the highest value for grain yield/plant $53.827 \mathrm{~g}$, and some of its components such as number of spikes/plant, weight of spikes/plant and biological yield/plant with 30.176, 68.915 g and 157.762, respectively. The cross Kauz $\times$ Iba-95 exhibited maximum value for average spike weight, weight of grains/spike and harvest index $4.941 \mathrm{~g}, 3.695 \mathrm{~g}$ and 0.438, respectively. Regards to the parental performance at the same location the line parent Hasad produced the highest value for average spike weight, 1000-grain weight and harvest index $4.463 \mathrm{~g}, 53.468 \mathrm{~g}$ and 0.466 , respectively, but the tester parent Saber beg produced the maximum weight for grain yield/plant $34.493 \mathrm{~g}$ and most its components such as number of spikes/plant, weight of spikes/plant, spike length and biological yield/plant 26.167, $53.748 \mathrm{~g}, 12.420 \mathrm{~cm}$ and $162.153 \mathrm{~g}$, respectively. At the second location the cross Klal $\times$ Cham- 6 produced the highest value for grain yield/plant and number of spikes/plant reached $23.422 \mathrm{~g}$ and 9.333 spikes, respectively. The cross Hasad $\times$ Iba-95 produced the highest amount for average spike weight, weight of grains/spike and harvest index reached $4.540 \mathrm{~g}, 3.439 \mathrm{~g}$ and 0.541 , respectively. The 
line parent Hasad showed maximum value for weight of spikes/plant, average spike weight, weight of grains/spike, 1000-grain weight and biological yield/plant with 34.018, 4.783, 3.627, 58.691 and $125.960 \mathrm{~g}$, respectively. Maximum grain yield/plant recorded by the tester parent Cham- 6 with $21.185 \mathrm{~g}$.

Table 3. ANOVA table for the studied characters ( $1^{\text {st }}$ location upper value and $2^{\text {nd }}$ location lower value)

\begin{tabular}{|c|c|c|c|c|c|c|c|c|c|c|c|}
\hline \multirow[b]{2}{*}{ S.O.V } & \multirow[b]{2}{*}{ d.f } & \multicolumn{10}{|c|}{ M.S } \\
\hline & & $\begin{array}{c}\text { No. of } \\
\text { spikes/plant }\end{array}$ & \begin{tabular}{|c|}
$\begin{array}{c}\text { Weight of } \\
\text { spikes/plant } \\
(\mathrm{g})\end{array}$ \\
\end{tabular} & \begin{tabular}{|c|} 
Average \\
spike \\
weight $(\mathrm{g})$ \\
\end{tabular} & $\begin{array}{c}\text { Spike } \\
\text { length }(\mathrm{cm})\end{array}$ & \begin{tabular}{|c|} 
No. of \\
grains/spike
\end{tabular} & $\begin{array}{c}\text { Weight of } \\
\text { grains/spike } \\
(\mathrm{g})\end{array}$ & $\begin{array}{l}1000 \text {-grain } \\
\text { weight }(\mathrm{g})\end{array}$ & $\begin{array}{c}\text { Biological } \\
\text { yield/plant }\end{array}$ & $\begin{array}{c}\text { Grain } \\
\text { yield/plant }\end{array}$ & $\begin{array}{l}\text { Harvest } \\
\text { index }\end{array}$ \\
\hline \multirow{2}{*}{ b } & & & 10.306 & 0.215 & 0.345 & 53 & 0.128 & 0 & & 63.330 & 0.005 \\
\hline & & 0.189 & 61.171 & 0.128 & 1.009 & 31.498 & 0.087 & 2.520 & 1214.901 & 6.308 & 0.003 \\
\hline \multirow{2}{*}{ enot } & & & $464.387 * *$ & $1.408 * *$ & $1.753 * *$ & $334.113 * *$ & $0.973 * *$ & $76.626 * *$ & $3192.694 * *$ & $246.838 * *$ & $0.019 * *$ \\
\hline & & 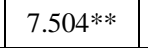 & $61.797 * *$ & $0.834 * *$ & $2.544 * *$ & $199.325^{* *}$ & $0.614 * *$ & $198.271 * *$ & $2232.995 * *$ & $21.414 * *$ & $0.038 * *$ \\
\hline \multirow{2}{*}{ Paren } & \multirow{2}{*}{6} & $*$ & $277.571 \mathrm{n} . \mathrm{s}$ & $0.842 * *$ & 0.95 & 2. & 0.65 & $5 * 3$ & 346 & $48.537 \mathrm{n} . \mathrm{s}$ & $0.030 * *$ \\
\hline & & 14.54 & $61.770 * *$ & $1.132 * *$ & $1.371^{* *}$ & $278.275^{* *}$ & $0.760 * *$ & $188.862 * *$ & $842.537 \mathrm{n} . \mathrm{s}$ & $3.422 \mathrm{n} . \mathrm{s}$ & $0.002 \mathrm{n} . \mathrm{s}$ \\
\hline & & & 298 & $0.238 \mathrm{n} . \mathrm{s}$ & 2.4 & 0.5 & 0.25 & 123 & 622 & $6 n$. & $0.002 \mathrm{n} . \mathrm{s}$ \\
\hline & & $4.035 \mathrm{n} . \mathrm{s}$ & $22.784 \mathrm{n} . \mathrm{s}$ & $0.017 \mathrm{n} . \mathrm{s}$ & $1.264 \mathrm{n} . \mathrm{s}$ & $22.060 \mathrm{n} . \mathrm{s}$ & $0.080 \mathrm{n} . \mathrm{s}$ & 5.125n.s 1 & $11351.405^{* * *}$ & $16.808^{*}$ & $0.159^{* * *}$ \\
\hline \multirow{2}{*}{$\mathrm{Cr}$} & & $102.426 * *$ & $581.323 * *$ & $1.822 * *$ & $2.121 * *$ & $418.760 * *$ & $1.212 * *$ & 78.119 ** & $3279.183 * *$ & $367.301 * *$ & 0.01 \\
\hline & & $3.982 *$ & 65.3 & $0.746^{*}$ & 3. & 17 & 0.5 & $* * 2$ & $k *$ & $* *$ & 0. \\
\hline \multirow{2}{*}{ Line } & \multirow{2}{*}{3} & $146.488 * *$ & $1530.861 * *$ & $2.121 *$ & $4.120 * *$ & $690.911^{*}$ & $1.160 *$ & $137.449 * 1$ & $10083.346^{* * *}$ & $994.871 * *$ & $0.006 \mathrm{n} . \mathrm{s}$ \\
\hline & & $0.546 \mathrm{n}$. & 56.173n.s & $0.125 \mathrm{n} . \mathrm{s}$ & $3.701 * *$ & $288.908^{*}$ & $0.035 \mathrm{n} . \mathrm{s}$ & $229.919 * 2$ & $2297.184 n . s$ & $34.161 *$ & o.02-11.5 \\
\hline \multirow{2}{*}{ Tester } & & $000 *$ & 197.865n.s & $4.721 * *$ & 1.749 & 711.784* & $3.510 * *$ & $110.988 \mathrm{n} . \mathrm{s}$ & 42.918n.s & $280.862 n . s$ & $0.032 *$ \\
\hline & & $9.215^{*}$ & $80.703 *$ & $1.834 *$ & $2.724 *$ & $26.134 n . s$ & $1.438 *$ & $440.316 * 3$ & 3039.766n.s & $35.245^{*}$ & $0.085^{* *}$ \\
\hline \multirow{2}{*}{$\mathrm{L} * \mathrm{~T}$} & \multirow{2}{*}{6} & $92.204 * *$ & $234.373 \mathrm{n} . \mathrm{s}$ & $0.707^{*}$ & $1.246 * *$ & $185.010 \mathrm{n} . \mathrm{s}$ & $0.472 \mathrm{n} . \mathrm{s}$ & 37.497n.s & 955.856n.s & $82.330 \mathrm{n} . \mathrm{s}$ & $0.013 * *$ \\
\hline & & $3.956^{*}$ & $64.835^{* *}$ & $0.694 *$ & $3.291^{* *}$ & $162.859^{*}$ & $0.571 * *$ & $143.365^{*}$ & $1802.699 *$ & $29.189 * *$ & $0.046^{* * *}$ \\
\hline \multirow{2}{*}{ Erro } & \multirow{2}{*}{36} & & 107. & 0.223 & . & (6.08 & 0.202 & 2 . & & 72.233 & 0.003 \\
\hline & & 1.508 & 13.375 & 0.278 & 0.331 & 51.654 & 0.147 & 44.161 & 673.454 & 3.901 & 0.004 \\
\hline
\end{tabular}

Data in Table 5 illustrate the estimation of heterosis values determined as the percentage of F1s deviation from mid parental value. The cross Aras $\times$ Iba-95 showed maximum positive heterosis values for grain yield/plant $129.087 \%$ and some its components such as number of spikes/plant, weight of spikes/plant and biological yield/plant reached $129.114,126.554$ and $119.050 \%$ respectively, while the cross Kauz $\times$ Saber beg showed maximum negative heterosis value for grain yield/plant $-54.917 \%$ and most its components such as weight of spikes/plant, average spike weight, spike length, number of grains/spike, weight of grains/spike and biological yield/plant reached $-59.244,-35.415,-10.034,-44.598,-38.727$ and $-53.409 \%$ respectively at the first location, while at the second location maximum positive heterosis for grain yield/plant was $18.137 \%$ recorded by the cross Klal $\times$ Cham -6 , but the cross Aras $\times$ Saber beg produced maximum positive heterosis for average spike weight, number of grains/spike and weight of grains/spike reached 19.432, 39.719 and $24.923 \%$, respectively. The cross Hasad $\times$ Saber beg produced maximum negative value for grain yield/plant $-41.316 \%$ and some its components such as weight of spikes/plant, average spike weight, weight of grains/spike and 1000-grain weight reached -51.048, -30.277, -34.955 and $-23.423 \%$, respectively. 
Table 4. Averages of studied characters for parents and their $F_{1}$ crosses $\left(1^{\text {st }}\right.$ location upper value and $2^{\text {nd }}$ location lower value)

\begin{tabular}{|c|c|c|c|c|c|c|c|c|c|c|}
\hline $\begin{array}{l}\text { Crosses } \\
\text { and } \\
\text { Parents }\end{array}$ & $\begin{array}{c}\text { No. of } \\
\text { spikes/plant }\end{array}$ & $\begin{array}{c}\text { Weight of } \\
\text { spikes/plant } \\
\text { (g) }\end{array}$ & $\begin{array}{c}\text { Average } \\
\text { spike } \\
\text { weight }(\mathrm{g})\end{array}$ & $\begin{array}{c}\text { Spike } \\
\text { length }(\mathrm{cm})\end{array}$ & $\begin{array}{c}\text { No. of } \\
\text { grains/spike }\end{array}$ & $\begin{array}{l}\text { Weight of } \\
\text { grains/spike } \\
\text { (g) }\end{array}$ & $\begin{array}{c}\text { 1000- } \\
\text { grain } \\
\text { weight } \\
\text { (g) }\end{array}$ & $\begin{array}{c}\text { Biological } \\
\text { yield/plant(g) }\end{array}$ & $\begin{array}{c}\text { Grain } \\
\text { yield/plant(g) }\end{array}$ & $\begin{array}{c}\text { Harvest } \\
\text { index }\end{array}$ \\
\hline \multirow{2}{*}{$1 \times 5$} & 30.167 & 68.915 & 4.545 & 12.433 & 67.867 & 3.378 & 49.851 & 157.762 & 53.827 & 0.362 \\
\hline & 8.000 & 31.132 & 3.747 & 8.400 & 64.200 & 2.989 & 46.566 & 42.358 & 20.407 & 0.489 \\
\hline \multirow{2}{*}{$1 \times 6$} & 14.667 & 48.114 & 4.465 & 12.300 & 68.267 & 3.252 & 48.912 & 146.752 & 40.226 & 0.277 \\
\hline & 9.000 & 26.958 & 3.405 & 9.367 & 50.533 & 2.584 & 51.102 & 79.728 & 15.782 & 0.202 \\
\hline \multirow{2}{*}{$1 \times 7$} & 15.000 & 44.165 & 4.011 & 13.100 & 68.867 & 2.886 & 41.912 & 121.195 & 33.655 & 0.312 \\
\hline & 8.833 & 22.960 & 3.591 & 11.367 & 69.533 & 2.720 & 39.540 & 67.511 & 17.510 & 0.274 \\
\hline \multirow{2}{*}{$2 \times 5$} & 15.500 & 45.955 & 4.714 & 13.261 & 63.167 & 3.345 & 53.710 & 100.730 & 33.517 & 0.331 \\
\hline & 6.833 & 19.775 & 4.540 & 9.367 & 59.867 & 3.439 & 57.385 & 48.059 & 16.983 & 0.541 \\
\hline \multirow{2}{*}{$2 \times 6$} & 15.500 & 54.517 & 4.789 & 13.200 & 59.000 & 3.547 & 61.422 & 116.190 & 41.957 & 0.358 \\
\hline & 9.167 & 28.958 & 4.333 & 10.267 & 51.600 & 3.354 & 66.413 & 61.356 & 20.737 & 0.339 \\
\hline \multirow{2}{*}{$2 \times 7$} & 23.167 & 49.395 & 3.453 & 12.000 & 49.733 & 2.321 & 47.470 & 124.278 & 33.105 & 0.278 \\
\hline & 8.167 & 15.612 & 2.667 & 10.400 & 49.400 & 1.818 & 36.963 & 67.733 & 12.207 & 0.198 \\
\hline \multirow{2}{*}{$3 \times 5$} & 10.167 & 31.282 & 4.941 & 12.200 & 67.800 & 3.695 & 54.415 & 56.940 & 24.360 & 0.438 \\
\hline & 6.000 & 17.997 & 3.729 & 10.133 & 53.200 & 2.778 & 52.581 & 43.049 & 16.231 & 0.385 \\
\hline \multirow{2}{*}{$3 \times 6$} & 12.833 & 37.498 & 4.331 & 12.367 & 57.333 & 3.312 & 58.090 & 73.510 & 25.387 & 0.357 \\
\hline & 9.333 & 24.983 & 3.980 & 10.933 & 64.867 & 3.056 & 47.225 & 116.105 & 22.735 & 0.196 \\
\hline \multirow{2}{*}{$3 \times 7$} & 11.167 & 17.980 & 2.537 & 10.700 & 32.133 & 1.727 & 53.629 & 65.773 & 14.285 & 0.217 \\
\hline & 9.167 & 26.847 & 3.148 & 11.667 & 53.867 & 2.388 & 44.900 & 43.736 & 20.818 & 0.483 \\
\hline \multirow{2}{*}{$4 \times 5$} & 17.333 & 27.690 & 3.353 & 11.067 & 52.000 & 2.493 & 48.914 & 97.763 & 23.667 & 0.247 \\
\hline & 9.000 & 26.582 & 3.648 & 11.633 & 68.267 & 2.592 & 38.076 & 115.053 & 20.943 & 0.230 \\
\hline \multirow{2}{*}{$4 \times 6$} & 12.167 & 29.710 & 3.542 & 12.033 & 51.067 & 2.511 & 49.971 & 61.643 & 23.797 & 0.372 \\
\hline & 9.333 & 27.860 & 3.923 & 11.500 & 66.867 & 3.027 & 45.269 & 100.662 & 23.422 & 0.243 \\
\hline \multirow{2}{*}{$4 \times 7$} & 9.833 & 32.382 & 3.009 & 11.113 & 40.800 & 2.095 & 51.066 & 95.213 & 18.985 & 0.207 \\
\hline & 6.833 & 22.897 & 3.538 & 9.833 & 65.333 & 2.593 & 41.114 & 67.688 & 18.512 & 0.273 \\
\hline \multirow{2}{*}{ Line 1} & 15.500 & 29.352 & 3.100 & 11.033 & 41.133 & 2.117 & 52.338 & 59.797 & 22.222 & 0.414 \\
\hline & 8.500 & 22.738 & 3.148 & 10.100 & 47.600 & 2.392 & 50.764 & 97.788 & 20.060 & 0.210 \\
\hline \multirow{2}{*}{ Line 2} & 10.833 & 34.978 & 4.463 & 11.367 & 60.267 & 3.218 & 53.468 & 87.040 & 28.780 & 0.466 \\
\hline & 8.167 & 34.018 & 4.783 & 10.900 & 62.200 & 3.627 & 58.691 & 125.960 & 20.572 & 0.175 \\
\hline \multirow{2}{*}{ Line 3} & 10.833 & 34.483 & 4.453 & 11.367 & 61.067 & 3.317 & 51.508 & 120.190 & 28.878 & 0.252 \\
\hline & 6.000 & 21.937 & 3.461 & 9.767 & 51.533 & 2.613 & 50.665 & 82.145 & 18.703 & 0.248 \\
\hline \multirow{2}{*}{ ine 4} & 17.333 & 24.616 & 3.677 & 12.400 & 58.267 & 2.555 & 43.873 & 115.332 & 25.085 & 0.223 \\
\hline & 9.000 & 22.447 & 3.706 & 11.167 & 64.867 & 2.778 & 42.848 & 77.243 & 18.467 & 0.243 \\
\hline \multirow{2}{*}{ Tester 5} & 10.833 & 31.486 & 4.165 & 11.367 & 69.533 & 3.088 & 44.944 & 84.245 & 24.771 & 0.293 \\
\hline & 8.500 & 25.257 & 3.923 & 11.667 & 75.400 & 2.778 & 36.563 & 111.882 & 19.863 & 0.184 \\
\hline \multirow{2}{*}{ Tester 6} & 15.667 & 42.536 & 3.618 & 12.067 & 51.733 & 2.576 & 51.624 & 129.536 & 29.417 & 0.230 \\
\hline & 8.500 & 23.673 & 3.675 & 11.100 & 56.000 & 2.753 & 49.100 & 104.700 & 21.185 & 0.202 \\
\hline \multirow{2}{*}{ Tester 7} & 26.167 & 53.748 & 3.404 & 12.420 & 54.933 & 2.319 & 42.182 & 162.153 & 34.493 & 0.218 \\
\hline & 13.333 & 29.765 & 2.866 & 10.300 & 51.933 & 1.963 & 37.847 & 102.673 & 21.030 & 0.218 \\
\hline \multirow{2}{*}{$\begin{array}{c}\text { LSD } \\
(\mathrm{p} \leq 0.05)\end{array}$} & 5.456 & 20.772 & 0.792 & 0.909 & 15.792 & 0.744 & 7.854 & 46.757 & 14.074 & 0.093 \\
\hline & 6.056 & 0.874 & 0.953 & 11.901 & 0.634 & 11.004 & 42.973 & 3.270 & 0.105 & 2.034 \\
\hline
\end{tabular}


Table 5. \% Heterosis values for F1 crosses ( $1^{\text {st }}$ location upper value and $2^{\text {nd }}$ location lower value)

\begin{tabular}{|c|c|c|c|c|c|c|c|c|c|c|}
\hline Crosses & $\begin{array}{c}\text { No. of } \\
\text { spikes/plant }\end{array}$ & \begin{tabular}{|c|}
$\begin{array}{c}\text { Weight of } \\
\text { spikes/plant } \\
\text { (g) }\end{array}$ \\
\end{tabular} & \begin{tabular}{|c|} 
Average \\
spike \\
weight $(g)$
\end{tabular} & $\begin{array}{c}\text { Spike } \\
\text { length }(\mathrm{cm})\end{array}$ & $\begin{array}{c}\text { No. of } \\
\text { grains/spike }\end{array}$ & \begin{tabular}{|c|}
$\begin{array}{c}\text { Weight of } \\
\text { grains/spike } \\
(\mathrm{g})\end{array}$ \\
\end{tabular} & $\begin{array}{l}1000 \text {-grain } \\
\text { weight }(\mathrm{g})\end{array}$ & $\begin{array}{c}\text { Biological } \\
\text { yield/plant }\end{array}$ & $\underset{\text { yield/plant }}{\text { Grain }}$ & $\begin{array}{c}\text { Harvest } \\
\text { index }\end{array}$ \\
\hline \multirow{2}{*}{$1 \times 5$} & 9.114 & 126.554 & 25.124 & 11.012 & 22.651 & 29.790 & 2.488 & 119.050 & 129.087 & 2.499 \\
\hline & -5.882 & 29.729 & 5.997 & -22.818 & 4.390 & 15.616 & 6.648 & -59.595 & 2.229 & 148.223 \\
\hline \multirow{2}{*}{$1 \times 6$} & 882 & 859 & 32.917 & 6.494 & 47.021 & 38.580 & -5.905 & 55.020 & 55.799 & -13.886 \\
\hline & 5.882 & 16.171 & -0.195 & -11.635 & -2.445 & 0.454 & 2.343 & -21.251 & -23.474 & -1.859 \\
\hline \multirow{2}{*}{$1 \times 7$} & -28.000 & 6.294 & 23.350 & 11.711 & 43.373 & 30.117 & -11.317 & 9.209 & 18.681 & -1.108 \\
\hline & -19.084 & -12.539 & 19.432 & 11.438 & 39.719 & 24.923 & -10.756 & -32.644 & -14.772 & 28.093 \\
\hline \multirow{2}{*}{$2 \times 5$} & 43.077 & 38.285 & 9.256 & 16.666 & -2.671 & 6.094 & 9.153 & 17.617 & 25.177 & -12.868 \\
\hline & -18.000 & -33.277 & 4.296 & -16.987 & -12.984 & 7.390 & 20.489 & -59.587 & -15.997 & 201.578 \\
\hline \multirow{2}{*}{$2 \times 6$} & 16.981 & 40.663 & 18.528 & 12.660 & 5.357 & 22.441 & 16.891 & 7.297 & 44.189 & 2.972 \\
\hline & 10.000 & 0.390 & 2.467 & -6.667 & -12.690 & 5.141 & 23.226 & -46.800 & -0.679 & 79.505 \\
\hline \multirow{2}{*}{$2 \times 7$} & 25.225 & 11.342 & -12.228 & 0.897 & -13.657 & -16.176 & -0.743 & -0.256 & 4.641 & -18.771 \\
\hline & -24.031 & -51.048 & -30.277 & -1.887 & -13.435 & -34.955 & -23.423 & -40.749 & -41.316 & 0.508 \\
\hline \multirow{2}{*}{$3 x$} & -6.154 & -5.163 & 14.650 & 7.331 & 3.828 & 15.362 & 12.833 & -44.295 & -9.187 & 60.636 \\
\hline & -17.241 & -23.729 & 1.011 & -5.443 & -16.176 & 3.054 & 20.562 & -55.626 & -15.831 & 78.378 \\
\hline \multirow{2}{*}{$3 \times 6$} & -3.145 & -2.627 & 7.310 & 5.548 & 1.655 & 12.398 & 12.651 & -41.127 & -12.903 & 48.374 \\
\hline & 28.736 & 9.552 & 11.547 & 4.792 & 20.645 & 13.902 & -5.328 & 24.279 & 13.993 & -13.037 \\
\hline \multirow{2}{*}{$3 \times 7$} & -39.640 & -59.244 & -35.415 & -10.034 & -44.598 & -38.727 & 14.482 & -53.409 & -54.917 & -7.660 \\
\hline & -5.172 & 3.852 & -0.495 & 16.279 & 4.124 & 4.371 & 1.456 & -52.671 & 4.790 & 107.439 \\
\hline \multirow{2}{*}{$4 x$} & 23.077 & -1.287 & -14.481 & -6.872 & -18.623 & -11.626 & 10.146 & -2.029 & -5.059 & -4.393 \\
\hline & 2.857 & 11.446 & -4.361 & 1.898 & -2.662 & -6.695 & -4.103 & 21.669 & 9.279 & 7.656 \\
\hline \multirow{2}{*}{$4 \times 6$} & -26.263 & -11.514 & -2.892 & -1.635 & -7.152 & -2.131 & 4.653 & -49.652 & -12.675 & 64.480 \\
\hline & 6.667 & 20.815 & 6.296 & 3.293 & 10.645 & 9.474 & -1.534 & 10.652 & 18.137 & 9.064 \\
\hline \multirow{2}{*}{$4 \times 7$} & -46.847 & -24.017 & -20.504 & -6.558 & -34.440 & -22.515 & 17.224 & -22.716 & -35.931 & -19.035 \\
\hline & -37.405 & -16.772 & 4.233 & -10.470 & 2.618 & 9.408 & 10.507 & -36.904 & -9.464 & 35.543 \\
\hline \multirow{2}{*}{ S.E } & 13.844 & 13.677 & 6.152 & 2.771 & 7.986 & 7.080 & 3.357 & 14.339 & 14.425 & 9.266 \\
\hline & 5.534 & 7.036 & 3.443 & 3.447 & 4.722 & 4.445 & 4.131 & 12.584 & 5.307 & 25.955 \\
\hline
\end{tabular}

Table 6 clarified the general combining ability effect of parents. Combining ability plays important role in the estimation of inbred in terms of their breeding values, and this will help to decide efficient breeding method to be applied in segregating generation. Data in the same table revealed that the line parent Aras recorded significant and positive gca effect for grain yield and most its components viz. number of spikes/plant, weight of spikes/plant, number of grains/spike and biological yield/plant, while the tester parent Iba-95 gave significant and positive gca effect for average spike weight, weight of grains/spike and harvest index. Most negative gca effect values for the characters recorded by line parent Kauz and tester parent Saber beg at the first location. At the second location the highest positive and significant gca effect values for grain yield/plant and some its components such as spike length, number of grains/spike and biological yield/plant recorded by the line parent Klal, but the tester parent Cham-6 exhibited the highest positive and significant gca values for number of spikes/plant, 
weight of spikes/plant and weight of grains/spike. Maximum negative gca effect values for most characters recorded by the line parent Hasad and the tester parent Saber beg.

Table 6. Estimation of gca effect for the parents ( $1^{\text {st }}$ location upper value and $2^{\text {nd }}$ location lower value)

\begin{tabular}{c|c|c|c|c|c|c|c|c|c|c}
\hline Parents & $\begin{array}{c}\text { No. of } \\
\text { spikes/plant }\end{array}$ & $\begin{array}{c}\text { Weight of } \\
\text { spikes/plant }\end{array}$ & $\begin{array}{c}\text { Average } \\
\text { spike weight }\end{array}$ & $\begin{array}{c}\text { Spike } \\
\text { length }\end{array}$ & $\begin{array}{c}\text { No. of } \\
\text { grains/spike }\end{array}$ & $\begin{array}{c}\text { Weight of } \\
\text { grains/spike }\end{array}$ & $\begin{array}{c}\text { 1000-grain } \\
\text { weight }\end{array}$ & $\begin{array}{c}\text { Biological } \\
\text { yield/plant }\end{array}$ & $\begin{array}{c}\text { Grain } \\
\text { yield/plant }\end{array}$ & $\begin{array}{c}\text { Harvest } \\
\text { index }\end{array}$ \\
\hline \multirow{2}{*}{1} & 4.319 & 13.098 & 0.366 & 0.463 & 11.831 & 0.292 & -4.722 & 40.424 & 12.005 & 0.004 \\
& 0.306 & 2.637 & -0.106 & -0.694 & 1.628 & -0.014 & -1.525 & -7.887 & -0.958 & 0.001 \\
\hline \multirow{2}{*}{2} & 2.431 & 9.322 & 0.344 & 0.672 & 0.797 & 0.191 & 2.587 & 12.254 & 5.629 & 0.009 \\
& -0.250 & -2.932 & 0.159 & -0.394 & -6.172 & 0.092 & 6.326 & -12.037 & -2.215 & 0.038 \\
\hline \multirow{2}{*}{3} & -4.236 & -11.714 & -0.038 & -0.392 & -4.081 & 0.031 & 3.765 & -36.071 & -9.220 & 0.024 \\
& -0.139 & -1.104 & -0.068 & 0.506 & -2.483 & -0.038 & 0.974 & -3.456 & 1.071 & 0.034 \\
\hline \multirow{2}{*}{4} & -2.514 & -10.706 & -0.673 & -0.743 & -8.547 & -0.514 & -1.630 & -16.606 & -8.414 & -0.038 \\
& 0.083 & 1.399 & 0.015 & 0.583 & 7.028 & -0.041 & -5.775 & 23.381 & 2.102 & -0.073 \\
\hline \multirow{2}{*}{ S.E } & 1.098 & 4.181 & 0.159 & 0.183 & 3.179 & 0.150 & 1.581 & 9.412 & 2.833 & 0.019 \\
lines & 0.409 & 1.219 & 0.176 & 0.192 & 2.396 & 0.128 & 2.215 & 8.650 & 0.658 & 0.021 \\
\hline \multirow{2}{*}{5} & 2.667 & 2.827 & 0.414 & 0.092 & 6.206 & 0.348 & 0.109 & 1.820 & 3.279 & 0.031 \\
& -0.847 & -0.509 & 0.229 & -0.522 & 1.589 & 0.171 & 1.391 & -8.957 & -0.216 & 0.090 \\
\hline \multirow{2}{*}{6} & -1.833 & 1.826 & 0.308 & 0.327 & 2.414 & 0.275 & 2.985 & -1.955 & 2.278 & 0.028 \\
& 0.903 & 2.810 & 0.223 & 0.111 & -1.328 & 0.227 & 5.241 & 18.376 & 1.812 & -0.076 \\
\hline \multirow{2}{*}{7} & -0.833 & -4.653 & -0.722 & -0.420 & -8.619 & -0.623 & -3.094 & 0.136 & -5.556 & -0.060 \\
& -0.056 & -2.301 & -0.451 & 0.411 & -0.261 & -0.398 & -6.632 & -9.419 & -1.595 & -0.014 \\
\hline \multirow{2}{*}{ S.E } & 0.951 & 3.621 & 0.138 & 0.158 & 2.753 & 0.130 & 1.369 & 8.151 & 2.453 & 0.016 \\
& 0.354 & 1.056 & 0.152 & 0.166 & 2.075 & 0.111 & 1.918 & 7.491 & 0.570 & 0.018 \\
\hline \multirow{2}{*}{ testers }
\end{tabular}

Data represent in Table 7 explain the sca effect of the $\mathrm{F}_{1}$ crosses at both locations, sca is mainly a function of dominance variance, it helps in the identification of superior cross combination of heterosis. At the first location the cross Aras $\times$ Iba-95 was the best specific combiner for number of spikes/plant, weight of spikes/plant, biological yield/plant and grain yield/plant, while the cross Kauz $\times$ Iba-95 was the better specific combiner for average spike weight, number of grains/spike, weight of grains/spike and harvest index. At the second location the cross Aras $\times$ Iba- 95 was the best specific combiner for grain yield/plant, while the cross Hasad $\times$ Iba-95 was the best specific combiner for average spike weight, weight of grains/spike and harvest index. The cross Kauz $\times$ Cham- 6 was the best specific combiner for number of grains/spike and biological yield/plant.

Some genetic parameters present in Table 8 for both locations. At the first location the variance of sca effect was greater than those of gca effect for all characters except biological yield/plant and grain yield/plant, indicating to the importance of non additive gene effect in the inheritance of these characters, but for biological yield/plant and grain yield/plant the additive gene effect is more important in the inheritance of both. At the second location it was indicated that the magnitude of sca variance is larger than those of gca effect for all characters, confirming the importance of non additive gene effect in the inheritance of these characters. The average degree of dominance values are more than one for most characters at both locations. 
Table 7. Estimation of sca effect for the F1 crosses ( $1^{\text {st }}$ location upper value and $2^{\text {nd }}$ location lower value)

\begin{tabular}{|c|c|c|c|c|c|c|c|c|c|c|}
\hline Crosses & $\begin{array}{c}\text { No. of } \\
\text { spikes/plant }\end{array}$ & $\begin{array}{c}\text { Weight of } \\
\text { spikes/plant }\end{array}$ & $\begin{array}{c}\text { Average } \\
\text { spike } \\
\text { weight } \\
\end{array}$ & $\begin{array}{l}\text { Spike } \\
\text { length }\end{array}$ & $\begin{array}{c}\text { No. of } \\
\text { grains/spike }\end{array}$ & $\begin{array}{c}\text { Weight of } \\
\text { grains/spike }\end{array}$ & $\begin{array}{c}1000- \\
\text { grain } \\
\text { weight }\end{array}$ & $\begin{array}{c}\text { Biological } \\
\text { yield/plant }\end{array}$ & $\underset{\text { yield/plant }}{\text { Grain }}$ & $\begin{array}{c}\text { Harvest } \\
\text { index }\end{array}$ \\
\hline \multirow{2}{*}{$1 \times 5$} & 7.556 & 12.357 & -0.209 & -0.270 & -6.672 & -0.142 & 2.850 & 14.039 & 7.979 & 0.014 \\
\hline & 0.236 & 4.624 & -0.063 & -0.789 & 1.189 & 0.053 & -0.561 & -11.884 & 2.723 & 0.077 \\
\hline \multirow{2}{*}{$1 \times 6$} & -3.444 & -7.444 & -0.183 & -0.638 & -2.481 & -0.195 & -0.965 & 6.804 & -4.621 & -0.068 \\
\hline & -0.514 & -2.868 & -0.399 & -0.456 & -9.561 & -0.407 & 0.125 & -1.847 & -3.929 & -0.043 \\
\hline \multirow{2}{*}{$1 \times 7$} & -4.111 & -4.913 & 0.393 & 0.908 & 9.153 & 0.337 & -1.886 & -20.844 & -3.358 & 0.055 \\
\hline & 0.278 & -1.755 & 0.462 & 1.244 & 8.372 & 0.354 & 0.436 & 13.731 & 1.206 & -0.034 \\
\hline \multirow{2}{*}{$2 \times 5$} & -5.222 & -6.827 & -0.019 & 0.348 & -0.339 & -0.074 & -0.600 & -14.822 & -5.955 & -0.023 \\
\hline & -0.375 & -1.165 & 0.465 & -0.122 & 4.656 & 0.398 & 2.407 & -2.034 & 0.557 & 0.092 \\
\hline \multirow{2}{*}{$2 \times 6$} & -0.722 & 2.735 & 0.163 & 0.053 & -0.714 & 0.201 & 4.236 & 4.413 & 3.486 & 0.008 \\
\hline & 0.208 & 4.700 & 0.264 & 0.144 & -0.694 & 0.256 & 7.585 & -16.070 & 2.283 & 0.056 \\
\hline \multirow{2}{*}{$2 \times 7$} & 5.944 & 4.093 & -0.144 & -0.401 & 1.053 & -0.127 & -3.636 & 10.410 & 2.469 & 0.015 \\
\hline & 0.167 & -3.535 & -0.729 & -0.022 & -3.961 & -0.654 & -9.992 & 18.103 & -2.840 & -0.148 \\
\hline \multirow{2}{*}{$3 \times 5$} & -3.889 & -0.465 & 0.590 & 0.352 & 9.172 & 0.436 & -1.072 & -10.287 & -0.263 & 0.069 \\
\hline & -1.319 & -4.770 & -0.119 & -0.256 & -5.700 & -0.134 & 2.955 & -15.624 & -3.481 & -0.060 \\
\hline \multirow{2}{*}{$3 \times 6$} & 3.278 & 6.752 & 0.087 & 0.284 & 2.497 & 0.126 & -0.273 & 10.058 & 1.765 & -0.008 \\
\hline & 0.264 & -1.102 & 0.138 & -0.089 & 8.883 & 0.088 & -6.252 & 30.099 & 0.995 & -0.083 \\
\hline \multirow{2}{*}{$3 \times 7$} & 0.611 & -6.287 & -0.677 & -0.636 & -11.669 & -0.561 & 1.345 & 0.230 & -1.503 & -0.061 \\
\hline & 1.056 & 5.872 & -0.020 & 0.344 & -3.183 & 0.046 & 3.297 & -14.474 & 2.486 & 0.143 \\
\hline \multirow{2}{*}{$4 \times 5$} & 1.556 & -5.064 & -0.362 & -0.430 & -2.161 & -0.221 & -1.178 & 11.070 & -1.761 & -0.060 \\
\hline & 1.458 & 1.311 & -0.284 & 1.167 & -0.144 & -0.317 & -4.801 & 29.542 & 0.201 & -0.109 \\
\hline \multirow{2}{*}{$4 \times 6$} & 0.889 & -2.043 & -0.067 & 0.302 & 0.697 & -0.131 & -2.998 & -21.275 & -0.630 & 0.069 \\
\hline & 0.042 & -0.729 & -0.003 & 0.400 & 1.372 & 0.063 & -1.459 & -12.182 & 0.651 & 0.071 \\
\hline \multirow{2}{*}{$4 \times 7$} & -2.444 & 7.108 & 0.429 & 0.128 & 1.464 & 0.352 & 4.177 & 10.204 & 2.392 & -0.009 \\
\hline & -1.500 & -0.581 & 0.287 & -1.567 & -1.228 & 0.254 & 6.260 & -17.360 & -0.852 & 0.038 \\
\hline \multirow{2}{*}{ S. } & 1.902 & 7.242 & 0.276 & 0.317 & 5.506 & 0.259 & 2.738 & 16.302 & 4.907 & 0.032 \\
\hline & 0.709 & 2.111 & 0.305 & 0.332 & 4.149 & 0.221 & 3.837 & 14.983 & 1.140 & 0.036 \\
\hline
\end{tabular}

The simple correlation coefficient among studied characters represent in Table 9 at both locations. At the first location the character number of spikes/plant recorded positive and highly significant correlation with weight of spikes/plant, biological yield/plant and grain yield/plant 0.721, 0.709 and 0.681, respectively. Weight of spikes/plant recorded positive and highly significant correlation with spike length, biological yield/plant and grain yield/plant $0.644,0.760$ and 0.939 , respectively. Spike length exhibited positive and highly significant correlation with grain yield/plant 0.652 , but it recorded positive and significant correlation with number of grains/spike and weight of grains/spike 0.597 and 0.549 , respectively. Positive and highly significant correlation was recorded between number of grains/spike and each of grain weight/spike and grain yield/plant 0.849 and 0.609 , respectively. Positive and significant correlation was recorded between weight of grains/spike and each of harvest index and grain yield/plant with 0.531 and 0.551 , respectively. Positive and highly significant correlation was recorded between biological yield/plant and grain yield/plant 
0.755. At the second location the character number of spikes/plant correlated positively and significantly with weight of spikes/plant 0.492 , while weight of spikes/plant recorded positive and highly significant correlation with grain yield/plant 0.677. Positive and significant correlation recorded between spike length and biological yield/plant 0.487 . Positive and highly significant correlation was recorded between weight of grains/spike and 1000-grain weight 0.691 .

Table 8. Estimation of some genetic parameters $\left(1^{\text {st }}\right.$ location upper value and $2^{\text {nd }}$ location lower value)

\begin{tabular}{c|c|c|c|c|c|c|c|c|c|c}
\hline Parameters & $\begin{array}{c}\text { No. of } \\
\text { spikes/plantspikes/plant }\end{array}$ & $\begin{array}{c}\text { Weigh o } \\
\text { spike } \\
\text { weight }\end{array}$ & $\begin{array}{c}\text { Spike } \\
\text { length }\end{array}$ & $\begin{array}{c}\text { No. of } \\
\text { grains/spikegrains/spike }\end{array}$ & $\begin{array}{c}\text { Weigh o } \\
\text { grain } \\
\text { weight }\end{array}$ & $\begin{array}{c}\text { 1000- } \\
\text { iiological } \\
\text { yiant }\end{array}$ & $\begin{array}{c}\text { Grain } \\
\text { yield/plant }\end{array}$ & $\begin{array}{c}\text { Harvest } \\
\text { index }\end{array}$ \\
\hline$\sigma^{2} \mathrm{e}$ & 10.857 & 157.351 & 0.229 & 0.301 & 90.950 & 0.202 & 22.494 & 797.261 & 72.233 & 0.003 \\
& 1.508 & 13.375 & 0.278 & 0.331 & 51.654 & 0.147 & 44.161 & 673.454 & 3.901 & 0.004 \\
\hline \multirow{2}{*}{$\sigma^{2}$ gca } & 0.441 & 14.966 & 0.048 & 0.038 & 10.083 & 0.032 & 1.752 & 100.222 & 12.293 & 0.00007 \\
& 0.001 & 0.023 & 0.002 & 0.0004 & 0.411 & 0.0005 & 3.347 & 15.520 & 0.106 & 0.00005 \\
\hline \multirow{2}{*}{$\sigma^{2} \mathrm{sca}=\sigma^{2} \mathrm{D}$} & 27.115 & 25.674 & 0.159 & 0.315 & 31.353 & 0.090 & 5.001 & 52.860 & 3.366 & 0.003 \\
\hline \multirow{2}{*}{$\sigma^{2} \mathrm{gca} / \sigma^{2} \mathrm{sca}$} & 0.816 & 17.153 & 0.139 & 0.987 & 37.068 & 0.142 & 33.068 & 376.415 & 8.429 & 0.014 \\
\hline \multirow{2}{*}{$\sigma^{2} \mathrm{~A}$} & 0.016 & 0.583 & 0.302 & 0.120 & 0.322 & 0.354 & 0.350 & 1.896 & 3.653 & 0.023 \\
& 0.001 & 0.001 & 0.016 & 0.0004 & 0.011 & 0.003 & 0.101 & 0.041 & 0.013 & 0.003 \\
\hline \multirow{2}{*}{$\overline{\mathrm{a}}$} & 0.002 & 29.933 & 0.096 & 0.076 & 20.167 & 0.064 & 3.505 & 200.444 & 24.586 & 0.0001 \\
& 7.842 & 1.310 & 1.820 & 2.888 & 1.763 & 1.680 & 1.689 & 0.726 & 0.523 & 6.593 \\
\hline \multirow{2}{*}{$\mathrm{h}^{2} \mathrm{~b} . \mathrm{s}$} & 26.816 & 27.576 & 7.870 & 51.017 & 9.502 & 16.988 & 3.143 & 4.925 & 8.918 & 16.989 \\
\hline \multirow{2}{*}{$\mathrm{h}^{2} \mathrm{n} . \mathrm{s}$} & 0.721 & 0.261 & 0.528 & 0.564 & 0.362 & 0.433 & 0.274 & 0.241 & 0.279 & 0.520 \\
& 0.352 & 0.563 & 0.339 & 0.749 & 0.423 & 0.493 & 0.474 & 0.377 & 0.689 & 0.778 \\
\hline
\end{tabular}

Table 9. The simple correlation coefficient among all pairs of traits at both locations $\left(1^{\text {st }}\right.$ location upper value and $2^{\text {nd }}$ location lower value)

\begin{tabular}{|c|c|c|c|c|c|c|c|c|c|}
\hline Characters & $\begin{array}{c}\text { No. of } \\
\text { spikes/plant }\end{array}$ & \begin{tabular}{|c|}
$\begin{array}{c}\text { Weight of } \\
\text { spikes/plant } \\
(\mathrm{g})\end{array}$ \\
\end{tabular} & $\begin{array}{c}\text { Spike } \\
\text { length }(\mathrm{cm})\end{array}$ & \begin{tabular}{|c|} 
No. of \\
grains/spike
\end{tabular} & $\begin{array}{c}\text { Weight of } \\
\text { grains/spike(g) }\end{array}$ & \begin{tabular}{|c|}
$\begin{array}{c}1000- \\
\text { grain } \\
\text { weight }(g)\end{array}$ \\
\end{tabular} & $\begin{array}{c}\text { Biological } \\
\text { yield/plant }(g)\end{array}$ & $\begin{array}{c}\text { Harvest } \\
\text { index }\end{array}$ & $\begin{array}{c}\text { Grain } \\
\text { yield/plant(g) }\end{array}$ \\
\hline $\begin{array}{c}\text { No. of } \\
\text { spikes/plant }\end{array}$ & 1.000 & & & & & & & & \\
\hline \multirow{2}{*}{$\begin{array}{c}\text { Weight of } \\
\text { spikes/plant (g) }\end{array}$} & 0.721 & \multirow{2}{*}{1.000} & & & & & & & \\
\hline & 0.492 & & & & & & & & \\
\hline \multirow{2}{*}{$\begin{array}{c}\text { Spike } \\
\text { length }(\mathbf{c m})\end{array}$} & 0.345 & 0.644 & \multirow{2}{*}{1.000} & & & & & & \\
\hline & 0.312 & 0.047 & & & & & & & \\
\hline \multirow{2}{*}{$\begin{array}{c}\text { No. of } \\
\text { grains/spike }\end{array}$} & 0.120 & 0.467 & 0.597 & \multirow{2}{*}{1.000} & & & & & \\
\hline & -0.015 & 0.215 & 0.416 & & & & & & \\
\hline \multirow{2}{*}{$\begin{array}{c}\text { Weight of } \\
\text { grains/spike }(\mathrm{g})\end{array}$} & -0.071 & 0.424 & 0.550 & 0.850 & \multirow{2}{*}{1.000} & & & & \\
\hline & -0.313 & 0.381 & -0.061 & 0.372 & & & & & \\
\hline \multirow{2}{*}{$\begin{array}{l}\text { 1000-grain } \\
\text { weight (g) }\end{array}$} & -0.354 & -0.020 & 0.008 & -0.184 & 0.349 & \multirow{2}{*}{1.000} & & & \\
\hline & -0.311 & 0.203 & -0.365 & -0.401 & 0.691 & & & & \\
\hline \multirow{2}{*}{$\begin{array}{c}\text { Biological } \\
\text { yield/plant(g) }\end{array}$} & 0.709 & 0.760 & 0.431 & 0.356 & 0.127 & -0.415 & \multirow{2}{*}{1.000} & & \\
\hline & 0.384 & 0.358 & 0.487 & 0.278 & 0.052 & -0.182 & & & \\
\hline \multirow{2}{*}{ Harvest index } & -0.142 & 0.107 & 0.168 & 0.323 & 0.531 & 0.442 & -0.431 & \multirow{2}{*}{1.000} & \\
\hline & -0.299 & -0.061 & -0.399 & -0.067 & 0.261 & 0.300 & -0.819 & & \\
\hline \multirow{2}{*}{$\begin{array}{c}\text { Grain } \\
\text { yield/plant(g) }\end{array}$} & 0.681 & 0.939 & 0.652 & 0.609 & 0.551 & -0.031 & 0.756 & 0.179 & \multirow{2}{*}{1.000} \\
\hline & 0.400 & 0.677 & 0.351 & 0.335 & 0.357 & 0.097 & 0.479 & -0.073 & \\
\hline
\end{tabular}


Data represent in Table 10 illustrate the path coefficient analysis, indicating to the direct and indirect effects on grain yield. At first location the highest positive direct effect was recorded by number of grains/spike reached 0.947 and followed by 1000 -grain weight with 0.589 , while the highest negative direct effect recorded by weight of grains/spike reached -0.735 . The highest positive indirect effect was 0.805 recorded by number of grains/spike via weight of grains/spike and followed by number of grains/spike via spike length. The highest negative indirect effect in grain yield was -0.624 recorded by weight of grains/spike via number of grains/spike and followed by -0.403 for weight of grains/spike via spike length. At second location the highest positive direct effect in grain yield recorded by 1000-grain weight reached 1.425 and followed by number of grains/spike with 1.072, whilst only weight of grains/spike recorded negative direct effect reached -1.387 . Maximum positive indirect effect was 0.984 recorded by 1000 -grain weight via weight of grains/spike and the highest negative indirect effect was -0.958 recorded by weight of grains/spike via 1000-grain weight.

Table 10. Path coefficient analysis Illustrates direct effect (diagonal values) and indirect effect of studied characters in grain yield at both locations $\left(1^{\text {st }}\right.$ location upper value and $2^{\text {nd }}$ location lower value)

\begin{tabular}{|c|c|c|c|c|c|c|c|c|}
\hline Characters & $\begin{array}{c}\text { No. of } \\
\text { spikes/plant }\end{array}$ & $\begin{array}{c}\text { Weight of } \\
\text { spikes/plant } \\
\text { (g) }\end{array}$ & $\begin{array}{c}\text { Spike } \\
\text { length }(\mathbf{c m})\end{array}$ & $\begin{array}{c}\text { No. of } \\
\text { grains/spike }\end{array}$ & $\begin{array}{c}\text { Weight of } \\
\text { grains/spike(g) }\end{array}$ & $\begin{array}{l}\text { 1000-grain } \\
\text { weight (g) }\end{array}$ & $\begin{array}{c}\text { Biological } \\
\text { yield/plant(g) }\end{array}$ & $\begin{array}{c}\text { Harvest } \\
\text { index }\end{array}$ \\
\hline \multirow{2}{*}{$\begin{array}{c}\text { No. of } \\
\text { spikes/plant }\end{array}$} & 0.205 & 0.201 & -0.006 & 0.113 & 0.052 & -0.206 & 0.356 & -0.031 \\
\hline & 0.081 & 0.171 & 0.051 & -0.017 & 0.434 & -0.443 & 0.366 & -0.244 \\
\hline \multirow{2}{*}{$\begin{array}{c}\text { Weight of } \\
\text { spikes/plant } \\
\text { (g) } \\
\end{array}$} & 0.148 & 0.279 & -0.012 & 0.442 & -0.311 & -0.011 & 0.382 & 0.024 \\
\hline & 0.040 & 0.349 & 0.008 & 0.230 & -0.528 & 0.290 & 0.338 & -0.050 \\
\hline \multirow{2}{*}{$\begin{array}{c}\text { Spike } \\
\text { length }(\mathrm{cm})\end{array}$} & 0.071 & 0.180 & -0.018 & 0.565 & -0.404 & 0.005 & 0.216 & 0.037 \\
\hline & 0.025 & 0.016 & 0.164 & 0.446 & 0.085 & -0.520 & 0.461 & -0.327 \\
\hline \multirow{2}{*}{$\begin{array}{c}\text { No. of } \\
\text { grains/spike }\end{array}$} & 0.025 & 0.130 & -0.011 & 0.947 & -0.624 & -0.108 & 0.179 & 0.071 \\
\hline & -0.001 & 0.075 & 0.068 & 1.072 & -0.516 & -0.571 & 0.263 & -0.055 \\
\hline \multirow{2}{*}{$\begin{array}{c}\text { Weight of } \\
\text { grains/spike(g) }\end{array}$} & -0.014 & 0.118 & -0.010 & 0.805 & -0.735 & 0.206 & 0.064 & 0.117 \\
\hline & -0.025 & 0.133 & -0.010 & 0.399 & -1.387 & 0.984 & 0.049 & 0.214 \\
\hline \multirow{2}{*}{$\begin{array}{l}\text { 1000-grain } \\
\text { weight (g) }\end{array}$} & -0.073 & -0.005 & -0.0001 & -0.174 & -0.256 & 0.589 & -0.208 & 0.098 \\
\hline & -0.025 & 0.071 & -0.060 & -0.430 & -0.958 & 1.425 & -0.172 & 0.246 \\
\hline \multirow{2}{*}{$\begin{array}{c}\text { Biological } \\
\text { yield/plant }(g)\end{array}$} & 0.145 & 0.212 & -0.008 & 0.337 & -0.093 & -0.245 & 0.502 & -0.095 \\
\hline & 0.031 & 0.125 & 0.080 & 0.298 & -0.072 & -0.259 & 0.947 & -0.671 \\
\hline \multirow{2}{*}{ Harvest index } & -0.029 & 0.030 & -0.003 & 0.306 & -0.390 & 0.260 & -0.216 & 0.221 \\
\hline & -0.024 & -0.021 & -0.066 & -0.072 & -0.362 & 0.428 & -0.776 & 0.818 \\
\hline
\end{tabular}

\section{Discussion}

The results confirmed that the data for all characters under the study can be analyzed further to estimate general and specific combining ability effects. Similar results confirmed that both gca and sca variances were significant for most of the characters indicating importance of both additive as well as non additive components of genetic variance in the control of these traits (Kumar et al., 2011; Singh et al., 2013; Rahul, 2017). Also similar results reported by Burungale et al. (2011), Mandal et al. (2016), and Nataša et al. (2017). Highly significant differences among genotypes were recorded for all characters, also significant differences were recorded among the mean performance of $F_{1}$ s crosses (Fellahi et al., 2013; Kalhoro et al., 2015). Significant positive and negative heterosis for all characters were recorded, also significant positive 
relative heterosis for grain yield recorded previously by Ajmal et al. (2004), Akram et al. (2004), Akbar et al. (2009), Ashadusjman et al. (2012), Devi et al. (2013), Anonymous (2015), and Rahul (2017). The gca effect is primarily function of additive genetic variance, which helps in the selection of suitable good general combiner parent for hybridization (Rahul, 2017). These results are in confirmation with Kumar et al. (2011), Kapoor et al. (2011), Singh et al. (2013), Raj and Kandalkar (2013), Aslam et al. (2014), Ismail (2015), Kalhoro et al. (2015), and Kandil et al. (2016). According to Kenga et al. (2004) cross-combinations with high means, appropriate sca estimates and involving at least one of the parents with high gca would likely enhance the concentration of desirable genes to improve target traits. Present findings are in confirmation with Kumar et al. (2011), Kapoor et al. (2011), Singh et al. (2013), Raj and Kandalkar (2013), Aslam et al. (2014), Kalhoro et al. (2015), and Kandil et al. (2016). The average degree of dominance values are more than one for most characters at both locations. Previous reports confirmed the importance of over dominance in the inheritance of the grain weight/ spike of wheat which was pointed out by Petrović et al. (2012) and Adel and Ali (2013). Obverse to these results, the importances of partial dominance in the inheritance of this trait were reported by Kohan and Heidari (2014). However, the selection in early generations for the trait grain weight/spike would be difficult due to over-dominance type of gene action which indicates that the selection for this trait in advanced segregating generations would be more efficient (Nazir et al., 2014; Yao et al., 2014; Shehzad et al., 2015). While, partial dominance effect was observed in the inheritance of this trait reported by Farooq et al. (2011) and Rashid et al. (2013). Similar results, which indicated the importance of the dominant component in the genetic variance and over-dominant inheritance of the grain weight/ spike, have been also pointed out by El-Hosary et al. (2015). Converse to these results, the high value of the additive component and partial dominance responsible for the inheritance of grain weight/spike was observed by Minhas (2012) and Kohan and Heidari (2014). Heterosis estimates, for different morphological and yield related characters, are attributed to both additive and non additive gene actions. Heritability gives information about genetic variation; it is important for predicting the response to selection in the superseding generations. Heritability is dependent upon the type of gene action (Swati and Ramesh, 2004; Hasnain et al., 2006; Chowdhary et al., 2007). At the first location heritability in broad sense was high for number of spikes/plant, average spike weight, spike length and harvest index, while for the other characters it was low to moderate. At the second location it was high for spike weight/plant, spike length, grain yield/plant and harvest index, but for the rest it was low to moderate. Heritability in narrow sense was found to be low for almost all character at both locations. High heritability accompanied with high genetic advance shows that the heritability is due to additive genetic control and selection may be effective in early generations for these traits (Jamil et al., 2017). Heritability estimates varied from 54.48 to $80.91 \%$. Highest heritability $80.91 \%$ was observed for the cross combination Fareed $06 \times 9242$, While the lowest value of heritability $54.48 \%$ was estimated in cross combination Fareed $06 \times 9317$ (Waqas et al., 2014). Heritability for grain yield/plant was 0.68 in Kaleemullah et al. (2015), who said that this traits is greatly under genetic controlled and less influenced by environment. The estimates of heritability in narrow sense were medium to high reported by Yadav et al. (2011). Significant and positive correlation was recorded between grain yield and most its components. These results are harmony with those reported by Tofiq (2004), Hama-Ali (2006), Hama-Ameen (2008), Mohsin et al. (2009) 
and Fellahi et al. (2013). Estimation of the correlation between yield and its components alone is not enough to tell as the importance of each one of these components in determining the grain yield (Bhutta et al., 2005; Bhutto et al., 2005; Anwar et al., 2009; Ali and Shakor, 2012). Similar results are agreement with a positive correlation of grain yield with number of grains/spike and 1000-grain weight, with support the present studies (Belay et al., 1993; Aycecik and Yildirim, 2006). Positive and highly significant correlations recorded between grain yield/plant and harvest index reported by Tawfiq et al. (2016). The path analysis is used to find the direct or indirect effects of some yield components on grain yield in relation to yield with other components. Many researchers have been done on wheat breeding in which both correlation and path analysis methods were simultaneously used. Positive and significant correlation between plant height and grain yield were reported in many studies (Khayatnezhad et al., 2010; Zakizadeh et al., 2010; Amri et al., 2011). It was shown that seed numbers/spike has a positive effect on grain yield (Taleei and Bahram-Nejad, 2003; Foroozanfar et al., 2011). Path analysis conducted by a large number of researchers, it has been observed that 1000-grain weight has a positive effect on yield, followed by spike length, while grains/spike had a negative direct effect on grain yield associated a positive direct effect and negative indirect effect on grain yield via harvest index, while biological yield had the highest direct effect on grain yield refers to followed by weight of main spike, they show that the biological yield and weight of main spike can be used as criteria for selection to improve the yield of wheat grain (Mohammadi et al., 2007; Mollasadeghi et al., 2011; Iftikhar et al., 2012; Ayer et al., 2017).

\section{Conclusion}

Line parent Aras and tester parent Iba-95 was found to be a good general combiner for grain yield/plant at the first location, which produced a best specific combiner for this character, while at the second location the line parent Aras and the tester parent Iba95 were poor general combiner for grain yield/plant, whilst they produced the best specific combiner for this character. Low ratio of $\sigma^{2} \mathrm{gca} / \sigma^{2} \mathrm{sca}$ for most characters at the first location and for all characters at the second location and low estimates of heritability in narrow sense confirmed involvement of both additive and non additive gene effects with preponderance of non additive type of gene actions. The preponderance of non additive type of gene actions clearly indicated that selection of superior plants should be postponed to later generations.

Good general combine line parents like Aras and Klal for yield and most related components at both locations could be used in the development of high yielding varieties through selection for promising segregating cross generations. The crosses Aras $\times$ Iba-95 and Klal $\times$ Cham- 6 was found high yielding which were very desirable for further breeding program.

\section{REFERENCES}

[1] Adel, A. A., Ali, E. A. (2013): Gene action and combining ability in a six parent diallel cross of wheat. - Asian J. Crop Sci. 5(1): 14-23.

[2] Ajmal, S., Asif, M., Munir, M. (2004): Implication of combining ability: Analysis of some characteristics of spring wheat. - Quarterly Sci Vision 9(1-2): 1-5. 
[3] Akbar, M., Anwar, J., Hussain, M., Qureshi, M. H., Khan, S. (2009): Line $\times$ tester Analysis in bread wheat (Triticum aestivum L.). - J Agric Res 47(1): 411.

[4] Akram, Z., Ajmal, S. U., Khan, K. S., Qureshi, R., Zubair, R. (2004): Combining ability estimates of some yield and quality related traits in spring wheat (Triticum aestivum L.). - Pak J Bot 43(1): 221-231.

[5] Ali, I., Shakor, E. (2012): Heritability, variability, genetic correlation and path analysis for quantitative traits in durum and bread wheat under dry farming conditions. Mesopotamia Journal of Agriculture 40(4): 27-39.

[6] Altieri, M. A., Nicholls, C. I. (2017): The adaptation and mitigation of traditional agriculture in a changing climate. - Climatic Change 140: 33-45.

[7] Amri, M., Kazemi-Arbat, H., Rustaei, M. (2011): Relation Ships of some morphologic and physiologic traits with grain yield and its components in wheat (Triticum aestivum L.). - The $1^{\text {th }}$ national conference of new concepts in agriculture. Islamic Azad University. Savah branch.

[8] Anonymous (2015): Progress Report of All India Coordinated Wheat and Barley Improvement Project, 2014-2015. - IIW and BR, India, pp. 1: 1.

[9] Anwar, J., Ali, M., Hussain, M., Sabir, W., Khan, M., Zulkiffal, M., Abdullah, M. (2009): Assessment of yield criteria in bread wheat through correlation and path analysis. Journal of Animal and Plant Sciences 19(4): 185-188.

[10] Ashadusjman, M., Shamsuddoha, M., Alam, M. J., Begum, M. O. (2012): Combining ability and gene action for different root characters in spiring wheat. - J Env Sci and natural resources 5(2): 73-76.

[11] Aslam, R., Munawar, M., Salam, A. (2014): Genetic architecture of yield components accessed through line $\times$ tester analysis in wheat (Triticum aestivum L.). - Universal J PL Sci 2(5): 93-96.

[12] Aycecik, M., Yildirim, T. (2006): Path coefficient analysis of yield and yield components in bread wheat (Triticum aestivum L.) genotypes. - Pak J. Bot. 38(2): 417-424.

[13] Ayer, D. K., Shama, A., Ojha, B. R., Paudel, A., Dhakal, K. (2017): Correlation and path coefficient analysis in advanced wheat genotypes. - SAARC. J. Agri. 15(1): 1-12.

[14] Basbag, S., Ekinci, R., Gencer, O. (2007): Combining ability and heterosis for earliness characters in line $\mathrm{x}$ tester population of Gossypium hirsutum L. - Hereditas 144(5): 185190.

[15] Belay, S., Struik, P. C., Nachit, M. M., Peacock, J. M. (1993): Ontogenic analysis of yield components and yield stability of durum wheat in water- limited environments. Euphytica 71(3): 211-219.

[16] Bhutta, W. M., Akhtar, J., Anwar-ul-Haq, M., Ibrahim, M. (2005): Cause and effect relations of yield components in spring wheat (Triticum aestivum L.) under normal conditions. - Caderno de Pesquisa Sér. Bio., Santa Cruz do Sul. 17(1): 7-12.

[17] Bhutto, L. A., Soomro, Z. A., Ansari, B. A., Jarwar, A. R., Jalbani, B. H. (2005): Estimation of phenotypic correlation between grain yield and Its main components in Brassica species. - Indus J. of P Sci. 3(4): 327-331.

[18] Burungale, S. V., Chauhan, R. M., Gami, R. A., Thakor, D. M., Patel, P. T. (2011): Combining ability analysis for grain and quality traits in bread Wheat (Triticum aestivum L.). - Trends in Bio Science 4(1): 120-122.

[19] Chowdhary, M. A., Sajad, M., Ashraf, M. I. (2007): Analysis on combining ability of metric traits in bread wheat (Triticum aestivum L.). - Journal of Agricultural Research 45(1): 11-17.

[20] Curtis, B. (2002): Wheat in the world. - In: Curtis, B., Rajaram, S., Macpherson, H. (eds.) Bread wheat improvement and production. Food and Agricultural Organization of the United Nations, Rome.

[21] Devi, L., Swati, G. P., Singh, M., Jaiswal, J. P. (2013): Heterosis studies for yield and yield contributing traits in bread wheat (Triticum aestivum L.). - The Bioscan 8(3): 905909. 
[22] El-Hosary, A. A., Gehan, A., El-Deen, N. (2015): Genetic analysis in the F1 and F2 wheat generations of dilllel crosses. - Egypt. J. Plant Breed. 19(2): 355-373.

[23] Erkul, A., Unay, A., Konak, C. (2010): Inheritance of yield and yield components in bread wheat (Triticum aestivum L.) cross. - Turk. J. Field Crops 15(2): 137-140.

[24] FAO (2014): Statistical year book (2014). - Asia and the pacific food and agricultural.

[25] FAO (2016): Statistical yearbook (2016). - Area and production.

[26] Farooq, J., Khaliq, I., Kashif, M., Ali, Q., Mahpara, S. (2011): Genetic analysis of relative cell injury percentage and some yield contributing traits in wheat under normal and heat stress conditions. - Chil. J. Agr. Res. 71: 511-520.

[27] Fellahi, Z. E. A., Hannachi, A., Bouzerzour, H., Boutekrabt, A. (2013): Line $\times$ Tester Mating Design Analysis for Grain Yield and Yield Related Traits in Bread Wheat (Triticum aestivum L.). - Hindawi Publishing Corporation International Journal of Agronomy Volume 2013, Article ID 201851, 9 pages http://dx.doi.org/ $10.1155 / 2013 / 201851$.

[28] Fellahi, Z., Hannachi, A., Bouzerzour, H., Boutekrabt, A. (2013): Correlation between traits and path analysis coefficient for grain yield and other quantitative traits in bread wheat under semi arid conditions. - Journal of Agriculture and Sustainability 3(1): 16-26.

[29] Foroozanfar, M., Bihamta, M. R., Peyghambary, A., Zeynali, H. (2011): Evaluation of Bread Wheat Genotypes Under Normal and Water Stress Conditions SNPS for Agronomic Traits. - The Science of Sustainable Agriculture Journal 21: 33-46.

[30] Hama-Ali, E. O. (2006): Estimation of Heterosis and Heritability in Half Diallel Crossing in some Local Common Wheat (Triticum aestivum L.). - M.Sc. Thesis. College of Agriculture University of Sulaimani.

[31] Hama-Ameen, T. N. (2008): Full Diallel Crosses in Durum Wheat (Triticum durum). M.Sc. Thesis. College of Agriculture University of Sulaimani.

[32] Hasnain, Z., Abbas, G., Saeed, A., Shakeel, A., Muhammad, A., Rahim, M. A. (2006): Combining ability for plant height and yield related traits in wheat (Triticum aestivum L.). - Journal of Agricultural Research 44: 167-175.

[33] Iftikhar, R., Khaliq, I., Kashif, M., Ahmad, M., Ullah, S. (2012): Study of morphological traits affecting grain yield in wheat (Triticum aestivum L.) under field stress condition. Middle-East Journal of Scientific Research 11: 19-23.

[34] Iqbal, M., Navabi, A., Salmon, D. F., Yang, R. C., Murdoch, B. M., Moore, S. S., Spaner, D. (2007): Genetic analysis of flowering and maturity time in high latitude spring wheat: genetic analysis of earliness in spring wheat. - Euphytica 154(1-2): 207-218.

[35] Ismail, K. A. S. (2015): Heterosis and combining ability analysis for yield and its Components in Bread Wheat (Triticum aestivum L.). - Int J Curr Microbiol App Sci 4(8): $1-9$.

[36] Jain, S. K., Sastry, E. V. D. (2012): Heterosis and combining ability for grain yield and its contributing traits in bread wheat (Triticum aestivum L.). - RRJAAS 1: 17-22.

[37] Jamil, A., Khan, Sh., Sayal, O. U., Waqas, M., Ullah, Q., Ali, S. (2017): Genetic variability, broad sense heritability and genetic advance studies in bread wheat (Triticum aestivum L.) germplasm. - Pure Appl. Biol. 6(2): 538-543.

[38] Kahrizi, D., Cheghamirza, K., Kakaei, M., Mohammadi, R., Ebadi, A. (2010a): Heritability and genetic gain of some morpho-physiological variables of Durum wheat (Triticum turgidum var. durum). - Afr. J. Biotechnol. 9(30): 4687-4691.

[39] Kahrizi, D., Maniee, M., Mohammadi, R., Cheghamirza, K. (2010b): Estimation of genetic parameters related to morpho-agronomic traits of Durum wheat (Triticum turgidum var. durum). - Biharean Biologist 4(2): 93-97.

[40] Kaleemullah, I., Khalil, H., Subhan, F., Rabi, F., Bostan, N., Qureshi, S., Ahmad, W. (2015): Heritability, selection response and correlation for yield and yield components in irrigated wheat. - ARPN J. Agric. \& Biosci. 10(6): 217-225.

[41] Kalhoro, F. A., Rajpar, A. A., Kalhoro, Sh. A., Mahar, A., Ali, A., Otho, S. A., Soomro, R. N., Ali, F., Baloch, Z. A. (2015): Heterosis and Combing Ability in F1 Population of 
Hexaploid Wheat (Triticum Aestivum L.). - American Journal of Plant Sciences 6(7): 1011-1026.

[42] Kandil, A. A., Sharief, A. E., Gomaa, H. S. M. (2016): Estimation of general and specific combining ability in bread wheat (Triticum aestivum L.). - Int J Agri R 8(2): 37-44.

[43] Kapoor, E., Mondal, S., Dey, T. (2011): Combining ability analysis for yield and yield contributing traits in winter and spring wheat combinations. - J Wheat Res 3(1): 52-58.

[44] Kempthorne, O. (1957): An Introduction to Genetic Statistics. - John Wiley \& Sons, New York, NY, USA.

[45] Kenga, R., Alabi, S. O., Gupta, S. C. (2004): Combining ability studies in tropical sorghum (Sorghum bicolor (L.) Moench). - Field Crops Research 88(2-3): 251-260.

[46] Khan, M. A., Ahmad, N., Akbar, M., Rehman, A., Iqbal, M. M. (2007): Combining ability analysis in wheat. - Pak. J. Agri. Sci. 44(1): 1-5.

[47] Khayatnezhad, M., Zaefizadeh, M., Gholamin, R., Jamaati-e-Somarin, Sh. (2010): Study of Genetic Diversity and Path Analysis for Yield in Durum Wheat Genotypes under Water and Dry Condition. - World Applied Sciences Journal 9(6): 655-665.

[48] Kohan, M. Z., Heidari, B. (2014): Diallel cross study for estimating genetic components underlying wheat grain yield. - J. Biol. Environ. Sci. 8(22): 37-51.

[49] Kumar, A., Mishra, V. K., Vyas, R. P., Singh, V. (2011): Heterosis and combining ability analysis in bread wheat (Triticum aestivum L.). - J PL Breeding and Crop Sci 3(10): 209217.

[50] Kuzmanovic, L., Gennaro, A., Benedettelli, S., Dodd, I. C., Quarrie, S. A., Ceoloni, C. (2014): Structural-functional dissection and characterization of yield-contributing traits originating from a group 7 chromosome of the wheatgrass species Thinopyrum ponticum after transfer into durum wheat. - Journal of Experimental Botany 65: 509-525.

[51] Ljubičić, N., Petrović, S., Kostic, M., Dimitrijević, M., Hristov, N., Kondic-Spika, A., Jevtic, R. (2017): Diallel analysis of some important grain yield traits in bread wheat crosses. - Tukish Journal of Field Crops 22(1): 1-7.

[52] Mandal, A. B., Madhuri, G. (2016): Combining ability analysis for morphological and yield traits in wheat (Triticum aestivum L.). - J. Plant Sci. Res. 3(2): 157.

[53] Maniee, M., Kahrizi, D., Mohammadi, R. (2009): Genetic variability of some morphophysiological traits in Durum wheat (Triticum turgidum var. durum). - J. Appl. Sci. 9(7): 1383-1387.

[54] Minhas, N. M. (2012): Genetic Analysis for Grain Yield, Quality and Biochemical Traits in Wheat. - Ph.D. Dissertation, University of Rawalpindi, Pakistan.

[55] Mohammadi, A., Majidi, E., Bihamta, M. R. (2007): Heidari Sharifabad S. Evaluation of drought stress on agro - morphological characteristics in some wheat cultivars. Pajouhesh \& Sazandegi 73: 184-192.

[56] Mohsin, T., Khan, N., Naqvi, F. N. (2009): Heritability, phenotypic correlation and path coefficient studies for some agronomic characters in synthetic elite lines of wheat. - J. Food Agric. Environ 7(3-4): 278-282.

[57] Mollasadeghi, V., Imani, A. A., Shahryari, R., Khayatnezhad, M. (2011): Correlation and path analysis of morphological traits in different wheat genotypes under end drought stress condition. - Middle-East Journal of Scientific Research 7(2): 221-224.

[58] Moon, D. (2008): In the Russian Steppes: The Introduction of Russian Wheat on the Great Plains of the United States of America. - Journal of Global History 3: 203-225. http://dx.doi.org/10.1017/S1740022808002611.

[59] Nazir, A., Khaliq, I., Farooq, J., Mahmood, K., Mahmood, A., Hussain, M. M., Shahid, M. (2014): Pattern of inheritance in some yield related parameters in spring wheat (Triticum aestivum L.). - Am. J. Biol. and Life Sci. 2(6): 180-186.

[60] Petrović, S., Dimitrijević, M., Ljubičić, N., Banjac, B. (2012): Diallel analysis of quantitative traits in wheat crosses. - In: Proceedings of the 47th Croatian and 7 th International Symposium on Agriculture, Publisher University of Zagreb Faculty of Agriculture, Croatia, pp. 313-317. 
[61] Rahul, S. R. (2017): Combining Ability and Heterosis for Morpho-Physiological Characters on Bread Wheat (Triticum aestivum L.). - Agricultural Research \& Technology Open Access Journal. ISSN: 2471-6774.

[62] Raj, P., Kandalkar, V. S. (2013): Combining ability and heterosis analysis for grain yield and its components in wheat. - J Wheat Res 5(1): 45-49.

[63] Rashid, M. A. R., Khan, A. S., Iftikhar, R. (2013): Genetic studies for yield and yield related parameters in bread wheat. - Am. Eurasian J. Agric. Environ. Sci. 12(12): 15791583.

[64] Sehgal, D., Vikram, P., Sansaloni, C. P., Ortiz, C., Pierre, C. S., Payne, T., Ellis, M., Amri, A., Petroli, C. D., Wenzl, P., Singh, S. (2015): Exploring and mobilizing the gene bank biodiversity for wheat improvement. - PLoS ONE 10(7): e0132112. doi: 10.1371/journal.pone.0132112.

[65] Shehzad, M., Hussain, S. B., Qureshi, M. K., Akbar, M., Javed, M., Imran, H. M., Manzoor, S. A. (2015): Diallel cross analysis of plesiomorphic traits in Triticum aestivum L. genotypes. - Genet. Mol. Res. 14(4): 13485-13495.

[66] Shekhawat, U. S., Vijay, P., Singhania, D. L. (2001): Genetic divergence in barley (Hordeum vulgare L.). - Indian J. Agric. Res. 35(2): 121-123.

[67] Singh, R. K., Chaudhary, B. D. (1985): Biometrical Methods in Quantitative Genetic Analysis. - Kalyani, New Delhi, India.

[68] Singh, K., Singh, U. B., Sharma, S. N. (2013): Combining ability analysis for yield and its components in bread wheat (Triticum aestivum L.). - J Wheat Res 5(1): 63-67.

[69] Steel, R. G. D., Torrie, J. H. (1980): Principles and Procedures of Statistics: A Biometrical Approach. - McGraw Hill, New York, NY, USA.

[70] Swati, P. G., Ramesh, B. R. (2004): The nature and divergence in relation to yield traits in rice germplasm. - Annals of Agricultural Research 25: 598-602.

[71] Tahmasebi, S., Heidari, B., Pakniyat, H., Kamali, J., Reza, M. (2014): Independent and combined effects of heat and drought stress in the Seri M82× Babax bread wheat population. - Plant Breeding 133: 702-711.

[72] Taleei, A., Bahram-Nejad, B. (2003): A Study of Relationship Between Yield and Its Components in Landrace Populations of Wheat from Western Parts of Iran Using Multivariate Analysis. - Iranian Journal of Crop Sciences. Sci. 34(4): 949-959.

[73] Tawfiq, S. I., Haseeb, S., Abdulkhaleq, D. A., Hama, S. J. (2016): The Simple Correlation and Path Coefficient Analysis for Yield and Some Yield Components of Durum Wheat in Two Seasons in Iraqi Kurdistan. - Journal of Zankoy Sulaimani 18(3): 323-332.

[74] Tester, M., Langridge, P. (2010): Breeding technologies to increase crop production in a changing world. - Science 327: 818-822.

[75] Todorovska, E., Christov, N., Slavov, S., Christova, P., Vassilev, D. (2009): Biotic stress resistance in wheat breeding and genomic selection implications. - Biotechnol Biotec Eq. 23(4): 1417-1426.

[76] Tofiq, Sh. I. (2004): Partial Diallel Crossing in Common and Durum Wheat. - Ph.D. Dissertation Submitted to College of Agricultural University of Sulaimani.

[77] United Nations, Department of Economic and Social Affairs, Population Division, (2015): World Population Prospects: The 2015 Revision, Key Findings and Advance Tables. - Working Paper No. ESA/P/WP.241.

[78] Waqas, M., Faheem, M., Khan, A. S., Shazad, M., Ansari, M. A. A. (2014): Estimation of heritability and genetic advance for some yield traits in eight F2 populations of wheat (Triticum aestivum L.). - Sci. Lett. 2(2): 43-47.

[79] Wheat (2014): Wheat: Vital grain of civilization and food security. - In: 2013 Annual Report, CGIAR Research Program on Wheat. CGIAR Research Program on Wheat (Wheat), Mexico, D.F.

[80] Xiang, B., Li, B. (2001): A new mixed analytical method for genetic analysis of diallel data. - The Canadian Journal of Forest Research 31(12): 2252-2259. 
[81] Yadav, A. K., Maan, R. K., Kumar, S., Kumar, P. (2011): Variability, heritability and genetic advance for quantitative characters in hexaploid wheat (Triticum aestivum L.). Electronic Journal of Plant Breeding 2: 405-408.

[82] Yan, W., Hunt, L. A. (2002): Biplot analysis of diallel data. - Crop Science 42(1): 21-30.

[83] Yao, J., Ma, H., Yang, X., Yao, G., Zhou, M. (2014): Inheritance of grain yield and its correlation with yield components in bread wheat (Triticum aestivum L.). - Afr. J. Agric. Res. 13(12): 1379-1385.

[84] Zakizadeh, M., Esmaeilzadeh, M., Kahrizi, D. (2010): Study on genetic variation and relationship between plant characteristics and grain yield in long spike bread wheat (Triticum aestivum L.) genotypes-using multivariate analysis. - Iranian Journal of Crop Sciences (in Persian) 12(2): 18-30. 